\title{
SIZE AND LENGTH RELATIONS OF THE RIGHT AND LEFT TESTES OF PIGEONS IN HEALTH AND DISEASE
}

\author{
OSCAR RIDDLE \\ Carnegie Station for Experimental Evolution
}

The right ovary undergoes an early and more or less complete atrophy in most species of birds. Etzold ('91) has shown that in the sparrow the left testis is larger than the right. Firket ('14) and Swift ('15) have shown that in the chick embryo there were more primordial germ cells in the left gonad, and that this gonad is there also distinctly larger than the right. Allen ('07) found that the sex cells were unequally distributed to the two gonads of the turtle, the left receiving most. In this form only 24-70 per cent of the sex cells ever enter the gonads. Our own accumulation of data on the size and length relations of the two testes of young and adult pigeons show a very decided predominate number of larger right testes; and also a distinct difference in shape of the two glands-the left though actually smaller in size is usually absolutely longer than the right. Changes in the size relation in birds dead of certain diseases-particularly tuberculosis - and in hybrids are also suggested by our data

The meaning of this pronounced inequality in the distribution of the primordial germ cells which is plainly associated with a larger left embryonic gonad, and the finding in adults of two groups of birds of a marked and nearly constant larger gonad, but this a different gonad in the two cases, is by no means clear. But, whatever this may mean, it is probably a situation of importance to the theory of sex. We present our present data then with the confession that on the main points the meaning is not clear, but with the conviction that they are not less valuable because of our present inability to clarify the puzzling situation, and hopeful that the data may stimulate the further 
accumulation of facts from enough forms, and of such varied kinds, as may lead to a better understanding of the embryonic and adult inequalities of the sex glands of birds.

An examination of our data has shown that the measurements of glands of healthy birds should be grouped apart from those dead of disease; and those of pure species should be separated from hybrids. The justification of these separate groupings will appear later.

The relative size of the sex glands in healthy common pigeons

The weights of 31 pairs of testes from healthy common pigeons are recorded in table 1 . In 27 of these pairs the left testis was the smaller; in 4 the left was the larger. In two or three of these latter cases-12, 15 (22?) - the disparity of the two glands is so great as to make it clear that the smaller gland was wholly abnormal. In healthy common pigeons the right testis is larger than the left in a high proportion of cases.

Size relations of the testes of common pigeons dead of disease

In tables 2 and 6 the weights of 9 pairs of testes are given. In 7 of these the left gland was the smaller. It was larger in two instances; in one of these irregular cases, the smaller gland was again quite abnormally proportioned in reference to its larger associate

Size relations of testes of pure species, healthy and dead of disease

The testes of only 5 healthy birds of pure species (dead of cold, exposure, accident) are included in table 6 . In all of these cases the right testis was the larger.

The data for 46 individuals of pure species dead of disease are available. In table 2, 9 of the 10 individuals listed had larger right testes; the tenth had the two glands of equal size. Eleven further comparisons are supplied in table 3 . Of these, 7 right testes are larger, 2 are smaller, and 2 are the size-equivalents of the left. Table 6 gives the data for 30 additional pairs. Of these, 7 of St. risoria all had larger right testes; 2 of T. orientalis both had larger right testes; 13 of Spil. tigrina - mostly not 
mature birds had, 5 larger, 5 smaller, and 3 equivalent right testes. Four miscellaneous birds here had 2 larger and 2 smaller ${ }^{1}$ right testes.

TABLE 1

Weight of right and left testes of healthy common pigeons

\begin{tabular}{|c|c|c|c|c|c|c|c|}
\hline no. & DATE & WEIGHT & $\begin{array}{l}\text { PER CENT } \\
\text { OF DIFF. }\end{array}$ & no. & DATE & WEIGHT & $\begin{array}{l}\text { PER CENT } \\
\text { OF DIFF. }\end{array}$ \\
\hline 1 & April 5. & $\begin{array}{l}R=0.885 \\
L=0.515\end{array}$ & $-71.8^{*}$ & 17 & April $9 \ldots \ldots$ & $\begin{array}{l}\mathrm{R}=1.115 \\
\mathrm{~L}=1.015\end{array}$ & -9.9 \\
\hline 2 & April 5. & $\begin{aligned} \mathrm{R} & =1.475 \\
\mathrm{~L} & =0.845\end{aligned}$ & -746 & 18 & July $5 .$. & $\begin{array}{l}\mathrm{R}=1.010 \\
\mathrm{~L}=0.750\end{array}$ & -34.7 \\
\hline 3 & April 5. & $\begin{array}{l}\mathrm{R}=1.185 \\
\mathrm{~L}=0.990\end{array}$ & -19.7 & 19 & July 5. & $\begin{array}{l}\mathrm{R}=1.220 \\
\mathrm{~L}=1.375\end{array}$ & +12.7 \\
\hline 4 & April 5. & $\begin{array}{l}\mathrm{R}=1.055 \\
\mathrm{~L}=0.820\end{array}$ & -28.7 & 20 & July 5. & $\begin{array}{l}\mathrm{R}=1.140 \\
\mathrm{~L}=0.970\end{array}$ & -17.5 \\
\hline 5 & April 7. & $\begin{array}{l}\mathrm{R}=1.410 \\
\mathrm{~L}=0.765\end{array}$ & -84.3 & 21 & July 5. & $\begin{array}{l}\mathrm{R}=1.158 \\
\mathrm{~L}=0.765\end{array}$ & -51.4 \\
\hline 6 & April 7. & $\begin{array}{l}\mathrm{R}=1.190 \\
\mathrm{~L}=0.975\end{array}$ & -22.1 & 22 & July 5. & $\begin{array}{l}\mathrm{R}=0.370 \\
\mathrm{~L}=0.720\end{array}$ & +94.6 \\
\hline 7 & April 7. & $\begin{array}{l}\mathrm{R}=1.260 \\
\mathrm{~L}=1.000\end{array}$ & -26.0 & 23 & July 9. & $\begin{array}{l}R=0.855 \\
L=0.800\end{array}$ & -6.9 \\
\hline 8 & April 7. & $\begin{array}{l}\mathrm{R}=1.235 \\
\mathrm{~L}=0.945\end{array}$ & -30.7 & 24 & July 9. & $\begin{array}{l}\mathrm{R}=1.643 \\
\mathrm{~L}=1.030\end{array}$ & -59.5 \\
\hline 9 & April 7 . & $\begin{array}{l}\mathrm{R}=1.280 \\
\mathrm{~L}=1.225\end{array}$ & -4.5 & 25 & July 13. & $\begin{array}{l}\mathrm{R}=0.571 \\
\mathrm{~L}=0.540\end{array}$ & -5.7 \\
\hline 10 & April 9. & $\begin{array}{l}\mathrm{R}=1.075 \\
\mathrm{~L}=0.900\end{array}$ & -19.4 & 26 & July $13 \ldots$ & $\begin{array}{l}\mathrm{R}=0.820 \\
\mathrm{~L}=0.631\end{array}$ & -29.9 \\
\hline 11 & April 9. & $\begin{array}{l}\mathrm{R}=1.025 \\
\mathrm{~L}=0.710\end{array}$ & -44.4 & 27 & July 16 & $\begin{array}{l}\mathrm{R}=1.820 \\
\mathrm{~L}=1.500\end{array}$ & -21.3 \\
\hline 12 & April 9. & $\begin{array}{l}\mathrm{R}=0.025 \\
\mathrm{~L}=0.715\end{array}$ & +2760.0 & 28 & July 18 & $\begin{array}{l}\mathrm{R}=1.600 \\
\mathrm{~L}=0.536\end{array}$ & -198.5 \\
\hline 13 & April 9 & $\begin{array}{l}\mathrm{R}=1.460 \\
\mathrm{~L}=1.390\end{array}$ & -5.0 & 29 & July $20 \ldots$ & $\begin{array}{l}\mathrm{R}=0.051 \\
\mathrm{~L}=0.040\end{array}$ & -27.5 \\
\hline 14 & April 9 & $\begin{array}{l}\mathrm{R}=1.010 \\
\mathrm{~L}=0.720\end{array}$ & -40.3 & 30 & July 20 & $\begin{array}{l}R=1.390 \\
L=1.085\end{array}$ & -28.1 \\
\hline 15 & April 9. & $\begin{array}{l}\mathrm{R}=0.275 \\
\mathrm{~L}=1.425\end{array}$ & +418.2 & 31 & July 21 (juv.) & $\begin{array}{l}R=0.0004 \\
L=0.0003\end{array}$ & -33.3 \\
\hline 16 & April 9. & $\begin{array}{l}\mathrm{K}=1.125 \\
\mathrm{~L}=0.500\end{array}$ & -125.0 & Lef & 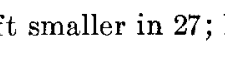 & larger in 4. & \\
\hline
\end{tabular}

* In calculating percentage differences in these tables the smaller gland is considered as equal to 100 per cent.

IIn both of these cases where the right testes weighed less than the left it will be seen that both testes were quite small-so small as perhaps to raise a question as to the reliability of the weights. 
Size of testes in healthy specific hybrids

In tables 4 and 5 the data for 30 healthy young hybrids are given. The very small gonad size of most of these young birds

TABLE 2

Weights of right and left testes of various pigeons (classified) dead of disease

\begin{tabular}{|c|c|c|c|c|c|c|}
\hline No. & DATE & wEIGHT & $\begin{array}{c}\text { PER CENT } \\
\text { OF DIFF. }\end{array}$ & DATE & WEIGET & $\begin{array}{l}\text { PER CENT } \\
\text { OF DIFF. }\end{array}$ \\
\hline
\end{tabular}

- 1. Common pigeons

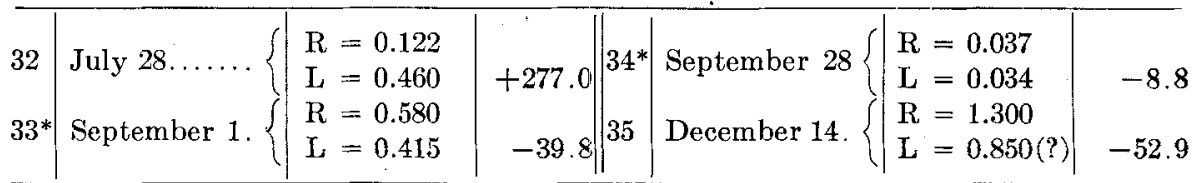

2. Blond and white wing doves and their hybrids

\begin{tabular}{|c|c|c|c|c|c|c|c|}
\hline \multicolumn{4}{|c|}{ Hybrids, (speeific) } & \multicolumn{4}{|c|}{ Pure } \\
\hline $36^{*}$ & June 10. & $\begin{array}{l}\mathrm{R}=0.417 \\
\mathrm{~L}_{3}=0.305\end{array}$ & -36.7 & $42 *$ & April $9 \ldots \ldots$ & $\begin{array}{l}\mathrm{R}=0.045 \\
\mathrm{~L}=0.025\end{array}$ & -80.0 \\
\hline $37 *$ & June 27 . & $\begin{array}{l}\mathrm{R}=0.050 \\
\mathrm{~L}=0.035\end{array}$ & -42.9 & $43^{*}$ & April $17 . .$. & $\begin{array}{l}\mathrm{R}=0.060 \\
\mathrm{~L}=0.050\end{array}$ & -20.0 \\
\hline $38 *$ & July 23. & $\begin{array}{l}\mathrm{R}=0.032 \\
\mathrm{~L}=0.046\end{array}$ & +43.8 & $44^{*}$ & September 26 & $\begin{array}{l}R=0.055 \\
L-0.055\end{array}$ & 0.0 \\
\hline 39 & September 12 & $\begin{array}{l}\mathrm{R}=0.765 \\
\mathrm{~L}=0.705\end{array}$ & -8.5 & $45^{*}$ & October 25. & $\begin{array}{l}\mathrm{R}=0.068 \\
\mathrm{~L}=0.056\end{array}$ & -21.4 \\
\hline 40 & October 17. & $\begin{array}{l}\mathbf{R}=0.0053 \\
\mathrm{~L}=0.0067\end{array}$ & +26.4 & 46 & October 25 & $\begin{array}{l}\mathbf{R}=0.046 \\
\mathrm{~L}=0.033\end{array}$ & -39.3 \\
\hline $41 *$ & October 31 & $\begin{array}{l}\mathrm{R}=0.090 \\
\mathrm{~L}=0.090\end{array}$ & $=0.0$ & $47 *$ & October 28 & $\begin{array}{l}\mathrm{R}=0.030 \\
\mathrm{~L}=0.027\end{array}$ & -11.1 \\
\hline
\end{tabular}

3. Other hybrids (speeific, ex. $51,52=$ gen.)

\begin{tabular}{|c|c|c|c|c|c|c|c|}
\hline 48 & August 28 . & $\begin{aligned} \mathrm{R} & =0.845 \\
\mathrm{~L} & =0.600\end{aligned}$ & -40.8 & $51 *$ & September $18 .\{$ & $\begin{array}{l}\mathrm{R}=0.580 \\
\mathrm{I}_{1}=0.510\end{array}$ & -13.7 \\
\hline 49 & June 10. & $\begin{array}{l}\mathbf{R}=0.190 \\
\mathbf{L}=0.160\end{array}$ & -18.8 & 52 & September 23. & $\begin{array}{l}\mathrm{R}=0.040 \\
\mathrm{~L}=0.031\end{array}$ & -29.0 \\
\hline 50 & August 28. & $\begin{array}{l}\mathrm{R}=0.133 \\
\mathrm{~L}=0.115\end{array}$ & -15.7 & $53^{*}$ & September $27 .\{$ & $\begin{array}{l}\mathrm{R}=0.010 \\
\mathrm{~L}=0.012\end{array}$ & +20.0 \\
\hline
\end{tabular}

4. Other pure species

\begin{tabular}{|c|c|c|c|c|c|c|c|}
\hline $54^{*}$ & August $26 \ldots$ & $\begin{array}{l}\mathrm{R}=0.040 \\
\mathrm{~L}=0.022\end{array}$ & -81.8 & 56 & October $31 \ldots$ & $\begin{array}{l}\mathrm{R}=0.465 \\
\mathrm{~L}=0.445\end{array}$ & -4.5 \\
\hline $55^{*}$ & November 10 & $\begin{array}{l}\mathrm{R}=0.033 \\
\mathrm{~L}=0.023\end{array}$ & -43.5 & $57^{*}$ & November 9. \{ & $\begin{array}{l}\mathrm{R}=0.015 \\
\mathrm{~L}=0.013\end{array}$ & -15.4 \\
\hline
\end{tabular}

\footnotetext{
* Tuberculosis found.
} 
TABLE 3 .

Weights of testes of doves (classified) dead of disease March 29 to November 25,1915

\begin{tabular}{|c|c|c|c|c|c|c|c|}
\hline no. & DATE & WEIGHT & $\begin{array}{l}\text { PER CENT } \\
\text { OF DIFF. }\end{array}$ & no. & DATE & WEIGHT & $\begin{array}{l}\text { PER CENT } \\
\text { OF DIFF. }\end{array}$ \\
\hline \multicolumn{4}{|c|}{ Pure species } & \multicolumn{4}{|c|}{ Specific hybrids } \\
\hline $59^{*}$ & March 29... & $\begin{array}{l}\mathrm{R}=0.012 \\
\mathrm{~L}=0.018\end{array}$ & +50.0 & $76^{*}$ & & $\begin{array}{l}\mathrm{R}=0.030 \\
\mathrm{~L}=0.031\end{array}$ & +3.3 \\
\hline $60^{*}$ & April $1 \ldots \ldots$ & $\begin{array}{l}\mathrm{R}=0.008 \\
\mathrm{~L}=0.005\end{array}$ & -60.0 & $77^{*}$ & July 23. & $\begin{array}{l}\mathrm{R}=0.262 \\
\mathrm{~L}=0.212\end{array}$ & -23.6 \\
\hline $61^{*}$ & April $2 \ldots .$. & $\begin{array}{l}\mathrm{R}=0.105 \\
\mathrm{~L}=0.105\end{array}$ & $=0.0$ & $78 *$ & July $30 .$. & $\begin{array}{l}\mathrm{R}=0.040 \\
\mathrm{~L}=0.032\end{array}$ & -25.0 \\
\hline $62^{*}$ & April $16 \ldots$ & $\begin{array}{l}\mathrm{R}=0.022^{1} \\
\mathrm{~L}=0.060\end{array}$ & +172.7 & $79^{*}$ & August 22. & $\begin{array}{l}\mathrm{R}=0.076 \\
\mathrm{~L}=0.077\end{array}$ & +1.3 \\
\hline 63 & September 2 & $\begin{array}{l}\mathrm{R}=0.158^{2} \\
\mathrm{~L}=0.146\end{array}$ & -8.2 & 80 & August 29. & $\begin{array}{l}\mathrm{R}=0.605^{2} \\
\mathrm{~L}=0.454\end{array}$ & -33.3 \\
\hline $64^{*}$ & September 5. & $\begin{array}{l}\mathrm{R}=0.032 \\
\mathrm{~L}=0.020\end{array}$ & -60.0 & $81^{*}$ & September 19 & $\begin{array}{l}\mathrm{R}=0.188 \\
\mathrm{~L}=0.152\end{array}$ & -23.7 \\
\hline 65 & $\begin{array}{c}\text { September } 11 \\
\text { (Juv.)...... }\end{array}$ & $\begin{array}{l}R=0.003 \\
L=0.002\end{array}$ & -50.0 & $82 *$ & September 28 & $\begin{array}{l}\mathrm{R}=0.026 \\
\mathrm{~L}=0.026\end{array}$ & $=0.0$ \\
\hline $66^{*}$ & September 14 & $\begin{array}{l}\mathrm{R}=0.023 \\
\mathrm{~L}=0.023\end{array}$ & $=0.0$ & $83^{*}$ & October 7 & $\begin{array}{l}\mathrm{R}=0.025 \\
\mathrm{~L}=0.021\end{array}$ & -19.0 \\
\hline $67^{*}$ & September 29 & $\begin{array}{l}\mathrm{R}=0.138 \\
\mathrm{~L}=0.116\end{array}$ & -18.9 & $84^{*}$ & $\begin{array}{l}\text { October } 19 \\
\text { (Juv.) } \ldots\end{array}$ & $\begin{array}{l}\mathrm{R}=0.007 \\
\mathrm{~L}=0.006\end{array}$ & -16.6 \\
\hline $68^{*}$ & October $3 \ldots$ & $\begin{array}{l}\mathrm{R}=0.045 \\
\mathrm{~L}=0.030\end{array}$ & -50.0 & $85^{*}$ & October $24 \ldots$ & $\begin{array}{l}\mathrm{R}=0.030 \\
\mathrm{~L}=0.030\end{array}$ & -0.0 \\
\hline $69^{*}$ & November $11 .\{$ & $\begin{array}{l}\mathrm{R}=0.082 \\
\mathrm{~L}=0.067\end{array}$ & -22.4 & $86 *$ & $\begin{array}{l}\text { November } 21 \\
\text { (Juv.) . . . }\end{array}$ & $\begin{array}{l}\mathbf{R}=0.015 \\
\mathbf{L}=0.020\end{array}$ & +33.3 \\
\hline \multicolumn{4}{|c|}{ Generic hybrids } & $87^{*}$ & & $\mathrm{~L}=0.016$ & +14.3 \\
\hline $70^{*}$ & April 2. & $\mathrm{R}=0.140$ & & 88 & November 25. & $\begin{array}{l}\mathrm{R}=0.018 \\
\mathrm{~L}=0.015\end{array}$ & -20.0 \\
\hline $71^{*}$ & May 30 & $\begin{array}{l}\mathrm{L}=0.125 \\
\mathrm{R}=0.320\end{array}$ & -12.0 & \multicolumn{4}{|c|}{ Common pigeons } \\
\hline 72 & June $20 \ldots$ & $\begin{array}{l}\mathrm{L}=0.280 \\
\mathrm{R}=0.082 \\
\mathrm{~L}=0.076\end{array}$ & -14.3 & 89 & April 28. & $R=1.260$ & \\
\hline $73^{*}$ & July $16 \ldots$ & $\begin{array}{l}\mathrm{R}=0.300 \\
\mathrm{~L}=0.298\end{array}$ & -0.7 & $90^{*}$ & August 25 . & $\begin{array}{l}L=1.035 \\
R=0.098 \\
L=0.087\end{array}$ & $\begin{array}{r}-21.7 \\
-12.6\end{array}$ \\
\hline $74^{*}$ & August 2 . & $\begin{array}{l}\mathrm{R}=0.012 \\
\mathrm{~L}=0.010\end{array}$ & -20.0 & 91 & April $5 \ldots \ldots\}$ & $\begin{array}{l}\mathrm{R}=1.105^{3} \\
\mathrm{~L}=0.990\end{array}$ & -11.6 \\
\hline $75^{*}$ & August 17 & $\begin{array}{l}\mathrm{R}=0.040 \\
\mathrm{~L}=0.037\end{array}$ & -8.1 & & & & \\
\hline
\end{tabular}

* Tuberculosis found.

1 The left suprarenal wholly involved in a tubercle nodule weighing nearly $1.0 \mathrm{gr}$.

2 Healthy.

${ }^{3}$ A hybrid from a family cross. 
TABLE 4

Weight, length and width of testes of young Ring dove (specific) hybrids-killed

\begin{tabular}{|c|c|c|c|c|c|}
\hline No. & DATE & WEIGHT & $\begin{array}{l}\text { PER CENT } \\
\text { OF } \\
\text { DIFFER- } \\
\text { ENCE }\end{array}$ & LENGTF AND WIDTH & $\begin{array}{c}\text { PER CENT } \\
\text { OF } \\
\text { DIFFER- } \\
\text { ENCE }\end{array}$ \\
\hline $88 a$ & December $4,1915$. & $\begin{array}{l}\mathrm{R}=0.035 \\
\mathrm{~L}=0.030\end{array}$ & -16.7 & $8.0 \times 3.8$ & \\
\hline $89 a$ & December $4,1915$. & $\begin{array}{l}\mathrm{R}=0.007(?) \\
\mathrm{L}=0.005(?)\end{array}$ & -40.0 & $\begin{array}{r}5.1 \times 1.6 \\
.6 \times 1.4\end{array}$ & -10.9 \\
\hline $90 a$ & December $4,1915$. & $\begin{array}{l}\mathrm{R}=0.004(?) \\
\mathrm{L}=0.005(?)\end{array}$ & +25.0 & $\begin{array}{l}4.1 \times 0.9 \\
4.9 \times 0.9\end{array}$ & +19.5 \\
\hline $91 a$ & December $4,1915$. & $\begin{array}{c}\mathrm{R}=\text { less than } \\
0.005(?) \\
\mathrm{L}=0.005(?)\end{array}$ & $+?$ & $\begin{array}{l}5.1 \times 0.8 \\
5.1 \times 1.5\end{array}$ & $=0.0$ \\
\hline 92 & December $4,1915$. & $\begin{array}{l}\mathrm{R}=0.005(?) \\
\mathrm{L}=0.005(?)\end{array}$ & $=0.0$ & $\begin{array}{l}4.8 \times 1.5 \\
5.3 \times 1.4\end{array}$ & +10.4 \\
\hline 93 & December 4,1915 & $\begin{array}{l}\mathrm{R}=0.007(?) \\
\mathrm{L}=0.005(?)\end{array}$ & -40.0 & $\begin{array}{l}4.8 \times 1.6 \\
5.3 \times 0.9\end{array}$ & +10.4 \\
\hline 94 & December 4, 1915 & $\begin{array}{l}\mathrm{R}=0.015 \\
\mathrm{~L}=0.010\end{array}$ & -50.0 & $\begin{array}{l}6.6 \times 1.9 \\
6.6 \times 1.5\end{array}$ & $=0.0$ \\
\hline $95^{*}$ & December $8,1915$. & $\begin{array}{l}\mathrm{R}=0.025 \\
\mathrm{~L}=0.025\end{array}$ & $=0.0$ & $\begin{array}{l}8.3 \times 2.2 \\
9.0 \times 1.9\end{array}$ & +8.4 \\
\hline 96 & December 8, 1915. . & $\begin{array}{l}\mathrm{R}=0.037 \\
\mathrm{~L}=0.025\end{array}$ & -48.0 & $\begin{array}{l}8.4 \times 2.7 \\
7.2 \times 2.2\end{array}$ & -16.7 \\
\hline 97 & December 8, 1915 & $\begin{array}{l}\mathrm{R}=0.010 \\
\mathrm{~L}=0.007\end{array}$ & -42.9 & $\begin{array}{l}5.0 \times 1.8 \\
5.0 \times 1.5\end{array}$ & $=0.0$ \\
\hline 98 & December 8, 1915. & $\begin{array}{l}R=0.320 \\
L=0.280\end{array}$ & -14.3 & & \\
\hline 99 & December 8, 1915. & $\begin{array}{l}\mathrm{R}=0.007(?) \\
\mathrm{L}=0.005(?)\end{array}$ & -40.0 & $\begin{array}{l}4.4 \times 1.7 \\
5.0 \times 1.0\end{array}$ & +13.6 \\
\hline 100 & December 8,1915 & $\begin{array}{l}\mathrm{R}=0.025 \\
\mathrm{~L}=0.020\end{array}$ & -25.0 & $\begin{array}{l}6.8 \times 2.2 \\
6.7 \times 2.1\end{array}$ & -1.5 \\
\hline 101 & December 8, 1915.. & $\begin{array}{l}\mathrm{R}=0.010 \\
\mathrm{~L}=0.007\end{array}$ & -42.9 & $\begin{array}{l}6.5 \times 1.9 \\
6.5 \times 1.5\end{array}$ & $=0.0$ \\
\hline 102 & December 8, 1915. & $\begin{array}{l}\mathrm{R}=0.012 \\
\mathrm{~L}=0.012\end{array}$ & $=0.0$ & \multirow{4}{*}{\multicolumn{2}{|c|}{$\begin{array}{lr}\text { Summary: } \\
\text { Left larger in.... } & 2 \\
\text { Left smaller in .... } & 13 \\
\text { Two equal in..... } & 3 \\
\text { Left longer in.... } & 5 \\
\text { Left shorter in ... } & 3 \\
\text { Left equal in..... } & 4\end{array}$}} \\
\hline 103 & December 8,1915 & $\begin{array}{l}\mathrm{R}=0.035 \\
\mathrm{~L}=0.025\end{array}$ & -40.0 & & \\
\hline 104 & December 8,1915 & $\begin{array}{l}\mathrm{R}=0.100 \\
\mathrm{~L}=0.070\end{array}$ & -42.9 & & \\
\hline 105 & December 8, 1915. & $\begin{array}{l}R=0.017 \\
L=0.015\end{array}$ & -13.3 & & \\
\hline
\end{tabular}

* Tuberculosis found. 
is probably responsible for the failure of our weighings to differentiate between the masses of several pairs of the testes. In the 30 pairs 18 right testes were larger, 5 were smaller, 7 were not differentiated by the weighings.

\section{Size of testes in hybrids dead of disease}

Seven of the 10 specific hybrids of table 2 had larger right testes; 2 had smaller; 1 had the testes of equal size. Of the two generic hybrids $(52,53)$ represented in this table, one had a larger and one a smaller right testes. In table 3 are listed 13 specific hybrids; 7 larger right testes, 4 smaller, and 2 equivalents.

TABLE 5

Weight, length and width of testes of young Ring dove (specific) hybrids-lilled

\begin{tabular}{|c|c|c|c|c|c|}
\hline No. & DATE & WEIGHT & $\begin{array}{l}\text { PER CENT } \\
\text { OF } \\
\text { DIFFER- } \\
\text { ENCE }\end{array}$ & LENGTH AND WIDTH & $\begin{array}{c}\text { PER CENT } \\
\text { OF } \\
\text { DIFFER- } \\
\text { ENCE }\end{array}$ \\
\hline 106 & November 29,1915 & $\mathrm{R}=\underset{\text { too small to }}{\text { weigh }}$ & & $3.2 \times 1.5$ & \\
\hline & & $\begin{array}{l}\mathrm{L}=0.005 \\
\mathrm{R}=0.007\end{array}$ & $+? .0$ & $\begin{array}{l}4.7 \times 1.8 \\
5.8 \times 1.6\end{array}$ & +46.9 \\
\hline 107 & November 29, 1915 & $\mathrm{~L}=0.007$ & $=0.0$ & $5.4 \times 2.0$ & -7.4 \\
\hline 108 & November 29,1915 & $\begin{array}{l}\mathrm{R}=0.009 \\
\mathrm{~L}=0.010\end{array}$ & +11.1 & $\begin{array}{l}5.8 \times 1.8 \\
6.4 \times 1.8\end{array}$ & +10.3 \\
\hline 109 & November 29,1915 & $\begin{array}{l}\mathrm{R}=0.020 \\
\mathrm{~L}=0.019\end{array}$ & -5.3 & $\begin{array}{l}8.2 \times 1.9 \\
8.2 \times 1.9\end{array}$ & $=0.0$ \\
\hline 110 & November 29,1915 & $\begin{array}{l}\mathrm{R}=0.018 \\
\mathrm{~L}=0.020\end{array}$ & +11.1 & $\begin{array}{l}7.4 \times 2.0 \\
7.4 \times 2.0\end{array}$ & $=0.0$ \\
\hline 111 & November 29,1915 & $\begin{array}{l}\mathrm{R}=0.010 \\
\mathrm{~L}=0.010\end{array}$ & $=0.0$ & $\begin{array}{l}5.9 \times 2.2 \\
7.4 \times 1.6\end{array}$ & +25.4 \\
\hline 112 & November 29,1915 & $\begin{array}{l}\mathrm{R}=0.190 \\
\mathrm{~L}=0.170\end{array}$ & -11.8 & $\begin{array}{l}17.0 \times 4.5 \\
15.7 \times 4.5\end{array}$ & -8.3 \\
\hline 113 & December $3,1915$. & $\begin{array}{l}\mathrm{R}=0.007 \\
\mathrm{~L}=0.005\end{array}$ & -40.0 & $\begin{array}{l}5.7 \times 1.5 \\
4.9 \times 0.9\end{array}$ & -16.3 \\
\hline 114 & December 3, 1915 . & $\begin{array}{l}\mathrm{R}=0.007 \\
\mathrm{~L}=0.005\end{array}$ & -40.0 & $\begin{array}{r}5.5 \times 1.4 \\
4.2 \times 1.0 \\
\end{array}$ & -30.9 \\
\hline 115 & December 3, 1915 . & $\begin{array}{l}R=0.007 \\
L=0.007\end{array}$ & $=0.0$ & \multirow{3}{*}{\multicolumn{2}{|c|}{$\begin{array}{l}\text { Summary: } \\
\text { Left larger in ...... } \\
\text { Left smaller in ..... } \\
\text { Two equal in ..... } \\
\text { Left longer in ..... } \\
\text { Left shorter in ..... } \\
\text { Two equal in...... }\end{array}$}} \\
\hline 116 & December 3, 1915. & $\begin{array}{l}R=0.005 \\
L=0.005\end{array}$ & $=0.0$ & & \\
\hline 117 & December 3,1915 & $\begin{array}{l}\mathrm{R}=0.007 \\
\mathrm{~L}=0.005\end{array}$ & -40.0 & & \\
\hline
\end{tabular}

THE ANATOMICAI, RECORD, VOL. 11 , No. 3 
TAELE 6

Weights and measurements of testes-birds classified as to kind and disease

\begin{tabular}{|c|c|c|c|c|c|c|}
\hline No. & DATE & DISEASE ${ }^{1}$ & WEIOHTS & $\begin{array}{c}\text { PER CENT } \\
\text { OF } \\
\text { DIFFER- } \\
\text { ENCE }\end{array}$ & $\begin{array}{l}\text { LENGTH AND } \\
\text { WIDTH }\end{array}$ & $\begin{array}{c}\text { PER } \\
\text { CENT OF } \\
\text { DIFFER- } \\
\text { ENCE }\end{array}$ \\
\hline \multicolumn{7}{|c|}{ Pure species-Spil. tigrina } \\
\hline 118 & January 7 & Worms .... & $\begin{array}{l}\mathrm{R}=\mathbf{0}=050 \\
\mathrm{~L}=0.050\end{array}$ & $=00$ & $\begin{array}{r}7.8 \times 3.4 \\
10.8 \times 3.0\end{array}$ & +385 \\
\hline 119 & January 9 & Worms (juv.). & $\begin{array}{l}\mathrm{R}=0.012 \\
\mathrm{~L}=0.014\end{array}$ & +16.6 & $\begin{array}{l}5.3 \times 2.2 \\
7.0 \times 1.8\end{array}$ & +32.1 \\
\hline 120 & January 11 & Liver.... & $\begin{array}{l}\mathrm{R}=0.040 \\
\mathrm{~L}=0.045\end{array}$ & +12.5 & $\begin{array}{l}7.3 \times 3.0 \\
9.0 \times 2.8\end{array}$ & +23.3 \\
\hline 121 & January 11 & Liver and worms & $\begin{array}{l}\mathrm{R}=0.055 \\
\mathrm{~L}=0.055\end{array}$ & $=0.0$ & $\begin{array}{l}8.3 \times 3.2 \\
8.3 \times 3.0\end{array}$ & $=0.0$ \\
\hline 122 & January 13 & Worms. & $\begin{array}{l}\mathrm{R}=0.080 \\
\mathrm{~L}=0.080\end{array}$ & $=0.0$ & $\begin{array}{r}9.3 \times 4.5 \\
10.6 \times 4.3\end{array}$ & +13.9 \\
\hline 123 & January 15 & Worms liver. & $\begin{array}{l}\mathrm{R}=0.040 \\
\mathrm{~L}=0.045\end{array}$ & +12.5 & $\begin{array}{l}6.5 \times 3.0 \\
8.2 \times 2.8\end{array}$ & +26.2 \\
\hline 124 & January 18 & Intest. and liver. & $\begin{array}{l}\mathrm{R}=0.031 \\
\mathrm{~L}=0.027\end{array}$ & -14.8 & $\begin{array}{l}5.1 \times 3.4 \\
6.7 \times 2.7\end{array}$ & +31.4 \\
\hline 125 & January 25 & Intest .... & $\begin{array}{l}\mathbf{R}=0.030 \\
\mathbf{L}=0.045\end{array}$ & +16.6 & $\begin{array}{l}7.1 \times 3.0 \\
8.6 \times 3.1\end{array}$ & +21.1 \\
\hline 126 & January 29 & Worms (old) & $\begin{array}{l}\mathbf{R}=0.030 \\
\mathbf{L}=0.022\end{array}$ & -36.4 & $\begin{array}{l}7.6 \times 2.6 \\
7.0 \times 2.3\end{array}$ & -8.6 \\
\hline 127 & March 1 & Intest. (juv.) & $\begin{array}{l}\mathbf{R}=0.004 \dagger \\
\mathbf{L}=0.004-\end{array}$ & $-? .0$ & $\begin{array}{l}4.2 \times 2.1 \\
4.4 \times 1.9\end{array}$ & +4.8 \\
\hline 128 & May 17 & Sp. Li. (old). & $\begin{array}{l}\mathbf{R}=0.047 \\
\mathrm{~L}=0.035\end{array}$ & -34.3 & $\begin{array}{l}8.7 \\
7.2\end{array}$ & -20.8 \\
\hline 129 & January 28 & Liver and spleen & $\begin{array}{l}\mathbf{R}=0.030 \\
\mathbf{L}=0.025\end{array}$ & -20.0 & $\begin{array}{l}6.8 \times 3.1 \\
7.2 \times 2.4\end{array}$ & $\begin{array}{r} \\
+5.9\end{array}$ \\
\hline 130 & January 28 & Worms.... & $\begin{array}{l}\mathrm{R}=0.027 \\
\mathrm{~L}=0.030\end{array}$ & +11.1 & $\begin{array}{l}6.7 \times 2.5 \\
8.8 \times 2.5\end{array}$ & +31.3 \\
\hline
\end{tabular}

Pure species-T. orientalis

\begin{tabular}{|c|c|c|c|c|c|c|}
\hline 131 & March 8 & Lu. liver. & $R=0.016$ & & & \\
\hline 132 & March 23 & Cold (juv.)... & $\begin{array}{l}\mathrm{R}=0.010 \\
\mathrm{~L}=0.008\end{array}$ & -25.0 & $\begin{array}{l}5.4 \times 1.5 \\
3.7 \times 1.7\end{array}$ & -45.9 \\
\hline 133 & March 23 & Cold (juv.). & $\begin{array}{l}\mathrm{R}=0.010 \\
\mathrm{~L}=0.008\end{array}$ & -25.0 & $\begin{array}{l}6.0 \times 1.4 \\
4.7 \times 1.7\end{array}$ & -27.6 \\
\hline 134 & March 23 & Cold (juv.). & $\begin{array}{l}\mathrm{R}=0.011 \\
\mathrm{~L}=0.009\end{array}$ & -22.2 & $\begin{array}{l}7.0 \times 1.4 \\
6.3 \times 1.5\end{array}$ & -11.1 \\
\hline 135 & March 25 & Intest. (juv.). & $\begin{array}{l}\mathrm{R}=0.010 \\
\mathrm{~L}=0.007\end{array}$ & -42.9 & $\begin{array}{l}5.8 \times 1.9 \\
5.5 \times 1.6\end{array}$ & -5.5 \\
\hline
\end{tabular}

1 Abbreviations of the names of organs to their first two letters, implies that advanced and very evident tuberculosis was found in those organs (lungs, spleen, liver, joints, mesentery, intestine). Where more than one organ was affected the name of the organ (or organs) apparently most affected is written first. When the word is written out it denotes that this organ was abnormal, but not necessarily tubercular; immature birds are designated-(juv.). 
TABLE 6-Continued

No. $\quad$ DATE $\quad$ DISEAGE $\quad$ WEIGHTS $\left|\begin{array}{c}\text { PER CENT } \\ \text { OF } \\ \text { DIFFER- } \\ \text { ENCE }\end{array}\right| \begin{gathered}\text { LENGTH AND } \\ \text { WIDTH }\end{gathered}$

Pure species-St, risoria

\begin{tabular}{|c|c|c|c|c|c|c|}
\hline 136 & December 4 & Sp. li. lu.. & $\begin{aligned} \mathrm{R} & =0.030\end{aligned}$ & -20.0 & & \\
\hline & & & $R=0.053$ & & $9.2 \times 3.3$ & \\
\hline 137 & January 29 & sp. 11.11. & $\begin{array}{l}\mathrm{L}=0.031 \\
\mathrm{R}=0.015\end{array}$ & -70.9 & $\begin{array}{r}10.5 \times 1.9 \\
6.0 \times 2.3\end{array}$ & +14.1 \\
\hline 138 & January 29 & Sp. lu. li. & $\mathrm{L}=0.010$ & -50.0 & $\begin{array}{l}6.6 \times 2.0 \\
84 \times 2.9\end{array}$ & +10.0 \\
\hline 139 & February 4 & Intest...... & $\begin{array}{l}\mathrm{R}=0.037 \\
\mathrm{~L}=0.020\end{array}$ & -85.0 & $8.8 \times 2.3$ & +4.8 \\
\hline 140 & March 15 & Lu. (?) liver. & $\begin{array}{l}\mathrm{R}=0.550 \\
\mathrm{~L}=0.401\end{array}$ & -37.1 & & \\
\hline 141 & May 10 & Sp... & $\begin{array}{l}\mathrm{R}=0.023 \\
\mathrm{~L}=0.019\end{array}$ & -21.1 & $\begin{array}{l}8.0 \\
8.0\end{array}$ & $=0.0$ \\
\hline 142 & May 14 & Jo., Iu. sp.; liver . & $\begin{array}{l}\mathrm{R}=0.020 \\
\mathrm{~L}=0.017\end{array}$ & -17.6 & $\begin{array}{l}6.7 \\
6.9 \\
\end{array}$ & +2.9 \\
\hline
\end{tabular}

Miscellaneous-pure species

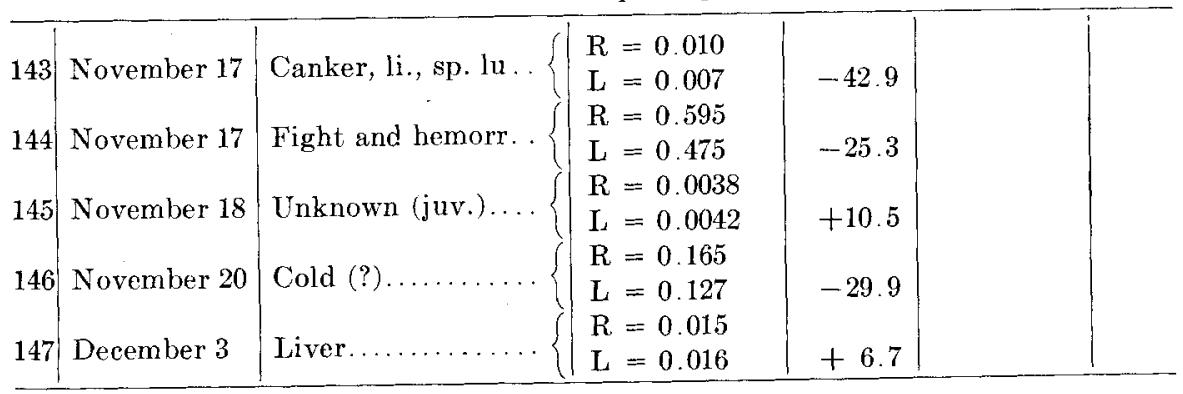

Common pigeons

\begin{tabular}{|c|c|c|c|c|c|c|}
\hline & & & $R=0.005(?)$ & & $5.4 \times 1.5$ & \\
\hline 148 & January 3 & Unknown (juv.). & $\mathrm{L}=0.005(?)$ & & $5.2 \times 1.3$ & -3.8 \\
\hline 149 & January 17 & Unknown (juv.). & $\begin{array}{l}\mathrm{R}=0.009(?) \\
\mathrm{L}=0.010(?) \\
\mathrm{R}=0.004 ?\end{array}$ & +11.1 & $\begin{array}{r}5.8 \times 1.5 \\
6.5 \times 1.7 \\
\text { about } 6.0\end{array}$ & +12.1 \\
\hline 150 & February 15 & Li. pleura. & $\mathrm{L}=0.003 ?$ & $-33.3 ?$ & $\begin{array}{l}\text { mm. long } \\
\text { about } 5.3 \\
\text { mm. long }\end{array}$ & +13.2 \\
\hline 151 & April 13 & Hemorrhage, liver.. & $\begin{array}{l}\mathrm{R}=0.014 \\
\mathrm{~L}=0.010\end{array}$ & -40.0 & $\begin{array}{l}6.4 \\
6.4\end{array}$ & $=0.0$ \\
\hline 152 & April 17 & Appar. healthy... & $\begin{array}{l}\mathrm{R}=1.340 \\
\mathrm{~L}=1.315\end{array}$ & -1.1 & $\begin{array}{l}20.2 \times 12.1 \\
23.9 \times 10.7\end{array}$ & +18.3 \\
\hline 153 & May 4 & Weakling (juv.).. & $\begin{array}{l}R=0.006 \\
L=0.004\end{array}$ & -50.0 & $\begin{array}{l}5.2 \\
5.2 \\
\end{array}$ & $=0.0$ \\
\hline
\end{tabular}


TABLE, 6-Continued

\begin{tabular}{|c|c|c|c|c|c|c|}
\hline xo. & DATE & DISEASE & WEIGHTS & $\begin{array}{l}\text { PER CENT } \\
\text { OF DIF- } \\
\text { FERENCE }\end{array}$ & $\begin{array}{l}\text { LENGTH } \\
\text { AND WIOTH }\end{array}$ & $\mid \begin{array}{c}\text { PER } \\
\text { CENT OF } \\
\text { MIFFER- } \\
\text { ENCE }\end{array}$ \\
\hline \multicolumn{7}{|c|}{ Hybrids-from crosses of species } \\
\hline 154 & January 1 & Cold? (juv.). & $\begin{array}{l}\mathrm{R}=0.000 \\
\mathrm{~L}=0.000\end{array}$ & & $\begin{array}{l}4.2 \times 0.9 \\
5.2 \times 1.2\end{array}$ & +23.8 \\
\hline 155 & January 4 & Sp. liver . . . . . . . & $\mathrm{R}=0.014$ & & $\begin{array}{l}6.3 \times 2.1 \\
86 \times 25\end{array}$ & \\
\hline & & & $\begin{array}{l}\mathrm{L}=0.018 \\
\mathrm{R}=0.000\end{array}$ & +21.4 & $\begin{array}{l}8.0 \times 2.3 \\
3.8 \times 1.5\end{array}$ & +36.0 \\
\hline 156 & January 8 & (Juv.)... & $I_{L}=0.000$ & & $5.7 \times 1.2$ & +50.0 \\
\hline 157 & January 15 & & $\begin{array}{l}\mathrm{R}=0.037 \\
\mathrm{~L}=0.035\end{array}$ & -5.7 & $\begin{array}{l}8.7 \times 2.7 \\
8.4 \times 2.8\end{array}$ & -3.6 \\
\hline 158 & December 3 & Li. (juv.).. & $\begin{array}{l}\mathrm{R}=0.003 \\
\mathrm{~L}_{1}=0.003\end{array}$ & $=0.0$ & & \\
\hline 159 & January 15 & Sp. jo............ & $\begin{array}{l}\mathrm{R}=0.027 \\
\mathrm{~L}=0.020\end{array}$ & -35.0 & & \\
\hline 160 & January 27 & $\begin{array}{l}\text { Healthy d wa rf; } \\
\text { killed............ }\end{array}$ & $\begin{array}{l}\mathrm{R}=0.010(?) \\
\mathrm{L}=0.013(?)\end{array}$ & $+30.0 ?$ & & \\
\hline 161 & February 18 & Sp. $1 \mathrm{u} \ldots \ldots \ldots \ldots$ & $\begin{array}{l}\mathrm{R}=0.015 \\
\mathrm{~L}=0.015\end{array}$ & $=0.0$ & & \\
\hline 162 & March 6 & Sp. li., ete. & $\begin{array}{l}\mathrm{R}=0.028 \\
\mathrm{~L}=0.025\end{array}$ & -12.0 & & \\
\hline 163 & January 22 & Li., sp..... & $\begin{array}{l}\mathrm{R}=0.007 \\
\mathrm{~L}=0.007\end{array}$ & $=0.0$ & $\begin{array}{l}5.0 \times 2.1 \\
5.6 \times 2.0\end{array}$ & +12.0 \\
\hline 164 & January 24 & Lu., liver, spleen... & $\begin{array}{l}\mathrm{R}=0.050 \\
\mathrm{~L}=0.050\end{array}$ & $=0.0$ & $\begin{array}{r}9.7 \times 2.9 \\
10.4 \times 2.7\end{array}$ & +7.2 \\
\hline 165 & January 27 & Liver, lu. (?) ...... & $\begin{array}{l}\mathrm{R}=0.052 \\
\mathrm{~L}=0.050\end{array}$ & --4.0 & $\begin{array}{r}9.0 \times 3.7 \\
11.4 \times 2.9\end{array}$ & +26.6 \\
\hline 166 & February 13 & $\begin{array}{l}\text { Lu., liver, spleen } \\
\quad(\text { juv.)............ }\end{array}$ & $\begin{array}{l}\mathrm{R}=0.002 ? \\
\mathrm{~L}=0.003+\end{array}$ & +50.0 & $\begin{array}{l}5.7 \\
5.9\end{array}$ & +3.5 \\
\hline 167 & March 17 & Sp., lu. liver........ & $\begin{array}{l}\mathrm{R}=0.022 \\
\mathrm{~L}=0.021\end{array}$ & -4.7 & & \\
\hline 168 & March 18 & Sp. li., lu. & $\begin{array}{l}\mathrm{R}=0.025 \\
\mathrm{~L}=0.025\end{array}$ & $=0.0$ & $\begin{array}{l}7.2 \\
7.6\end{array}$ & +5.6 \\
\hline 169 & March 26 & Intest. (?) (juv.). & $\begin{array}{l}R=0.002 \\
\mathrm{C}=0.002\end{array}$ & $=0.0$ & $\begin{array}{l}4.4 \\
3.9\end{array}$ & -12.8 \\
\hline 170 & March 28 & Sp. me. lungs. & $\begin{array}{l}\mathrm{R}=0.048 \\
\mathrm{~L}=0.042\end{array}$ & -14.3 & $\begin{array}{r}9.9 \times 3.7 \\
10.1 \times 2.3\end{array}$ & +2.0 \\
\hline 171 & March 29 & Li. sp., me. & $\begin{array}{l}\mathrm{R}=0.031 \\
\mathrm{~L}=0.035\end{array}$ & +129 & $\begin{array}{r}8.7 \times 2.9 \\
12.2 \times 2.2\end{array}$ & +40.2 \\
\hline 172 & April 12 & Cold, lungs. & $\begin{array}{l}\mathrm{R}=1.015 \\
\mathrm{~L}=0.888\end{array}$ & -14.3 & $\begin{array}{l}22.6 \times 9.0 \\
23.5 \times 8.2\end{array}$ & +3.9 \\
\hline 173 & April 20 & (Juv.). & $\begin{array}{l}\mathrm{R}=0.002(?) \\
\mathrm{L}=0.002(?)\end{array}$ & $=0.0$ & $\begin{array}{l}5.2 \\
5.4\end{array}$ & +3.8 \\
\hline
\end{tabular}


TABLE 6-Continued

\begin{tabular}{|c|c|c|c|c|c|c|}
\hline so. & DATE & DISEASE & WEIGHTS & $\begin{array}{l}\text { PER CENT } \\
\text { OF DIF- } \\
\text { FERENCE }\end{array}$ & $\begin{array}{c}\text { LENGTH } \\
\text { AND WIDTH }\end{array}$ & $\begin{array}{c}\text { PER } \\
\text { CENT OF } \\
\text { DIFFER- } \\
\text { ENCE }\end{array}$ \\
\hline
\end{tabular}

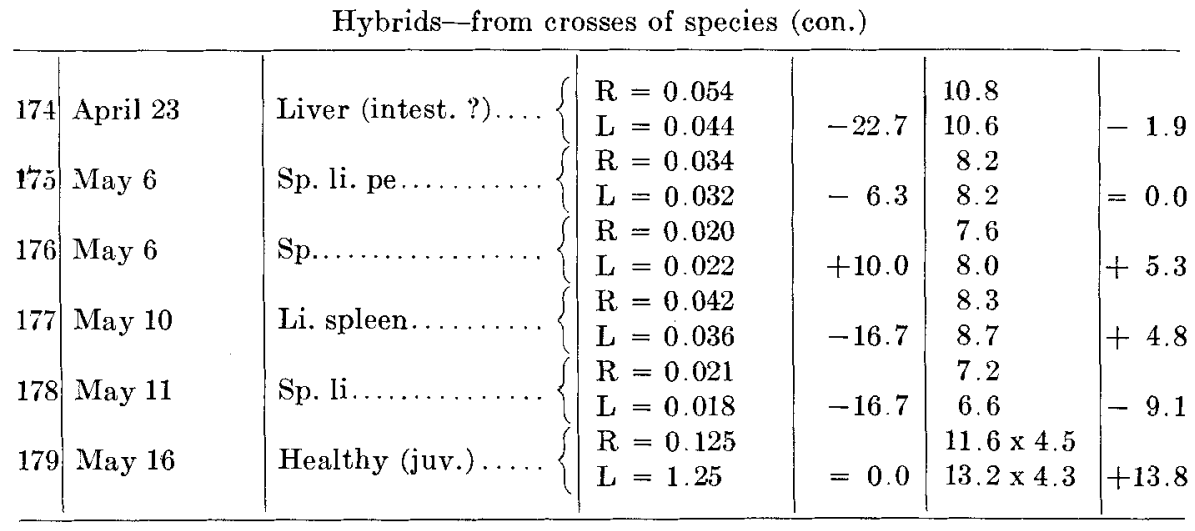

\begin{tabular}{|c|c|c|c|c|c|c|}
\hline \multicolumn{7}{|c|}{ Hybrids-from crosses of genera } \\
\hline 180 & December 4 & Sp., li......... & $\begin{array}{l}\mathrm{R}=0.017 \\
\mathrm{~L}=0.020\end{array}$ & +17.6 & $\begin{array}{l}7.0 \times 2.0 \\
6.3 \times 2.5\end{array}$ & -11.1 \\
\hline 181 & December 24 & Liver, pericard. & $\begin{array}{l}\mathrm{R}=0.017 \\
\mathrm{~L}=0.020\end{array}$ & +17.6 & & \\
\hline 182 & January 8 & Cold (?)..... & $\begin{array}{l}\mathrm{R}=0.005 \\
\mathrm{~L}=0.004\end{array}$ & -25.0 & $\begin{array}{l}3.2 \times 1.8 \\
3.0 \times 1.5\end{array}$ & -6.6 \\
\hline 183 & January 12 & Abdom, wall . & $\begin{array}{l}\mathrm{R}=0.009 \\
\mathrm{~L}=0.012\end{array}$ & +33.3 & $\begin{array}{l}7.3 \times 1.8 \\
5.7 \times 1.9\end{array}$ & -28.1 \\
\hline 184 & February 8 & Intest...... & $\begin{array}{l}\mathrm{R}=0.005 ? \\
\mathrm{~L}=0.004 ?\end{array}$ & -25.0 & $\begin{array}{l}\text { about } 4.5 \\
\text { about } 4.5\end{array}$ & $=0.0$ \\
\hline 185 & February 23 & Intest. (?) & $\begin{array}{l}\mathrm{R}=0.045 \\
\mathrm{~L}=0.051\end{array}$ & +12.3 & $\begin{array}{l}7.3 \\
7.1\end{array}$ & -0.3 \\
\hline 186 & March 7 & (Cold?) lungs . & $\begin{array}{l}\mathrm{R}=0.007 \\
\mathrm{~L}=0.005\end{array}$ & -40.0 & $\left\{\begin{array}{r}\text { both teste } \\
\text { gular-glo } \\
\text { like hem }\end{array}\right.$ & $\begin{array}{l}\text { s an- } \\
\text { bular, } \\
\text { p seed }\end{array}$ \\
\hline 187 & March 13 & Li., sp., lu. (?). & $\begin{array}{l}\mathrm{R}=0.014 \\
\mathrm{~L}=0.019\end{array}$ & +35.7 & $\begin{array}{l}7.0 \\
9.0\end{array}$ & +28.5 \\
\hline 188 & March 29 & $($ Cause? $) \ldots \ldots$ & $\begin{array}{l}\mathrm{R}=0.006 \\
\mathrm{~L}=0.007\end{array}$ & +16.7 & $\begin{array}{l}4.6 \times 1.9 \\
5.3 \times 2.0\end{array}$ & +15.2 \\
\hline 189 & April 12 & Worms, fighting. & $\begin{array}{l}\mathrm{R}=0.256 \\
\mathrm{~L}=0.212\end{array}$ & -20.8 & $\begin{array}{l}15.7 \times 5.6 \\
15.4 \times 5.1\end{array}$ & -1.9 \\
\hline 190 & May 15 & Lu......... & $\begin{array}{l}\mathrm{R}=0.047 \\
\mathrm{~L}=0.043\end{array}$ & -9.3 & $\begin{array}{l}8.8 \mathrm{x} \\
8.0 \mathrm{x}\end{array}$ & -10.0 \\
\hline
\end{tabular}


Six generic hybrids here all show larger right testes. Of 24 birds listed in table 6, larger rights were found in 11, smaller in 5, equivalents in 8 cases. In table 6,11 generic hybrids are listed; the right testis is larger in 5 , smaller in 6.

A summary representation of the weight relations of the testes of birds belonging to the several preceding groups is given in table 7 .

\section{Relative lengths of the two testes}

The length of 78 pairs of testes was ascertained. These were obtained from the testes of birds belonging to all of the groups discussed in the previous section of this paper. The reader is referred to the summary on 'length relations' given in table 7 for a first view of the result. Although a high proportion (126 to 39 for all groups) of the right testes are heavier, a reversal of proportions ( 24 to 42 ) is found for the absolute lengths of the two testes. In five of the seven groups of table 7 the left testis is absolutely longer. In one of the two exceptional groups-

TABLE 7

Summary of the preceding data

\begin{tabular}{|c|c|c|c|c|c|c|c|}
\hline \multirow{2}{*}{ CL_ASS } & \multirow{2}{*}{ STATE } & \multicolumn{3}{|c|}{ WEIGHT RELATIONSL } & \multicolumn{3}{|c|}{ TENCTXH RELATIONG } \\
\hline & & $L+$ & $L=$ & $I_{1}-$ & $L+$ & $\mathrm{L}=$ & $L-$ \\
\hline \multirow{2}{*}{ Pure species. . } & Healthy. & 0 & 0 & $\check{5}$ & 0 & 0 & 3 \\
\hline & Diseased. & $9^{2}$ & $7^{2}$ & 31 & 14 & 2 & 2 \\
\hline \multirow{2}{*}{ Common pigeons. } & Healthy. & 5 & 0 & 27 & 1 & 0 & 0 \\
\hline & Diseased. & 2 & 0 & 8 & 2 & 2 & 1 \\
\hline \multirow{2}{*}{ Specific hybrids . } & Healthy. . & 6 & 7 & 19 & 8 & 6 & 7 \\
\hline & Diseased. & 10 & 11 & 24 & 15 & 1 & 4 \\
\hline Generie hybrids. & Diseased. . & 7 & 0 & 12 & 2 & 1 & 6 \\
\hline \multicolumn{2}{|c|}{ 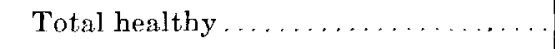 } & 11 & 7 & 51 & 9 & 6 & 10 \\
\hline \multicolumn{2}{|l|}{ Total diseased... } & 28 & 18 & 74 & 33 & 6 & 14 \\
\hline \multicolumn{2}{|l|}{ Grand total. } & 39 & 24 & 126 & 42 & 12 & 24 \\
\hline
\end{tabular}

1 The cases of Iarger left testis, arc grouped under L+; those of equal size under $\mathrm{L}=$; those with a smaller left testis under $\mathrm{L}-$.

${ }^{2}$ Five of the (9), and 3 of the (6), are from 14 Sp. tigrina-all dead at less than 9 months old. 
'healthy pure species'-it is possible that the smaller length of the left testis is connected with the nearest approximation to uniformity of smaller size in this group. In the second group-'diseased generic hybrids'- the testes show the greatest departure in their weight relations from what is elsewhere the rule.

An examination of the detailed measurements and percentage weight differences given in tables 4,5 and 6 is even more convincing than the summary of table 7 on the point that the two testes definitely tend to assume two different shapes-the left to be thinner and more elongate, the right to be shorter and thicker. This difference in form is perhaps not without interest since the only persistent gonad in the female-that of the left side--is characteristically 'thin' and 'long.' The testis that develops on this side is similarly characterized as compared with its mate of the right side.

Pure species and hybrids and the relative size of the two testes

Table 7 facilitates an expression of a relation which seems to obtain between degree of hydridization on the one hand, and the number of departures from the usual situation - a larger right and a smaller left testis-on the other. There are good reasons for believing that common pigeons-mongrels of many breeds probably not even descended from a single good species-may rightly occupy a place in this classification intermediate to specific hybrids and pure species. There is no question that the generic hybrids are more separated from the pure species than are the specific hybrids. Now, the number of gonad size relations, that depart from the rule, arrange themselves in all of the seven groups of the table, precisely in this order of departure from purity of species. That is, the greatest proportion of larger left testes is found in the group most widely separated from a pure species (generic hybrids); and the two intermediate stages of crossing (specific hybrids and common pigeons) show each its appropriately smaller intermediate number of larger left testes. 
The influence of disease on the actual and relative size of the two testes

The detailed data of the several tables and the summary of table 7 demonstrate that diseased birds furnish a higher proportion of larger left testes - violations of the more general rulethan do healthy birds. Further, the testes of pigeons suffer great reduction in several, or most, forms of disease. Now it probably happens that because of the great reduction in size of the testes in disease that this of itself has rendered the results of a few of the weighings of the smallest glands less certain. ${ }^{1}$ An examination of the detailed data will, however, leave no doubt that disease is a very real and important reason for the observed differences.

It has been found well to designate the cause of death in most of the tables. Advanced tuberculosis is easily; and with much certainty, diagnosed in pigeons. It is the most common cause of death among the birds of our collection. All deaths from this cause are so designated in the tables. A careful comparison of the size of the testes of birds dead of tuberculosis as compared with those dying of other or unknown causes will show that the gonads of the male suffer greatest reduction under this disease. This is certainly not tune for the female gonad; a point upon which data are still being collected. In this connection. Hatai's ('15) observation of the effects of exercise on the size of the ovary and testis of the rat are of interest. Hatai found a like qualitative response-an increase - in both; but quantitatively the response was quite different; the testes increased only 12.33 per cent while the increase in the ovaries was 84.33 per cent.

The several sections of table 6 show that in pigeons the spleen and liver are more often affected by tuberculosis than are other organs. It is the spleen too that suffers greatest hypertrophy and most complete transformation under the disease. This is true of both sexes.

${ }^{1}$ Because of jmperfect or anclean separation of the gland from body wall, and drying during weight; the greatest care wat not alwavs obtain perfect weights of the smaliest of these glands. 
Size relations of the testes in the common fowl

In table 8 are given the few data we have been able to obtain on the Jungle fowl, common fowl, and the duck. Since these were-with two exceptions - healthy fowls even the few data indicate a situation different from that found in pure species of pigeons. Whether these data are really representative of these forms, cannot now be determined. Whether hydridization (or mongrelization) of these forms is responsible for the apparent predominance of the reverse of the situation found in pigeons is doubtful. They like the sparrows may normally possess a larger left and a smaller right testis.

TABLE 8

Weights of testes of Jungle Fowl and common fowl

\begin{tabular}{|c|c|c|c|c|c|c|c|}
\hline No. & DATE & WEIGHT & $\begin{array}{l}\text { PER CENT } \\
\text { OF DIF- } \\
\text { FERENCE }\end{array}$ & no. & DATE & WEIGHT & $\begin{array}{l}\text { PER CENT } \\
\text { OF DIF } \\
\text { FERENCE }\end{array}$ \\
\hline \multicolumn{4}{|c|}{ Jungle fowl } & \multicolumn{4}{|c|}{ Common fowl } \\
\hline 1 & July $5 \ldots$ & $\mathrm{R}=3.203$ & +317 & 1 & July 16. & $\mathrm{R}=3.734$ & 100 \\
\hline 2 & July 5 . & $\begin{array}{l}\mathrm{R}=4.700 \\
\mathrm{~L}=5.700\end{array}$ & +21.3 & 2 & July 16 . & $\begin{array}{l}\mathrm{L}=5.880 \\
\mathrm{~L}=7.000\end{array}$ & $\begin{array}{l}+12.9 \\
+19.0\end{array}$ \\
\hline 3 & July 8. & $\begin{array}{l}\mathrm{R}=4.635 \\
\mathrm{~L}=4.280\end{array}$ & -8.4 & 3 & July 20 . & $\begin{array}{l}\mathrm{R}=6.540 \\
\mathrm{~L}=5.610\end{array}$ & -16.6 \\
\hline 4 & July $10 .$. & $\begin{array}{l}\mathrm{R}=6.305 \\
\mathrm{~L}=6.270\end{array}$ & -0.6 & 4 & July 22 . & $\begin{array}{l}R=11.660 \\
L=12.815\end{array}$ & $\begin{array}{r} \\
+9.9\end{array}$ \\
\hline 5 & July 12. & $\begin{array}{l}\mathrm{R}=4.500 \\
\mathrm{~L}=4.900\end{array}$ & $\begin{array}{r} \\
+8.9\end{array}$ & 5 & August 26 . & $\begin{array}{l}\mathrm{R}=8.820 \\
\mathrm{~L}=7.545\end{array}$ & -16.9 \\
\hline 6 & July $16\left(\right.$ inj. $\left.^{1}\right)$ & $\begin{array}{l}\mathrm{R}=3.960 \\
\mathrm{~L}=5.105\end{array}$ & +28.9 & 6 & April 21. & $\begin{array}{l}R=11.100 \\
L=10.350\end{array}$ & -7.2 \\
\hline \multirow[t]{2}{*}{7} & July 22 (inj. $\left.{ }^{1}\right)\{$ & $\begin{array}{l}R=1.665 \\
L=1.820\end{array}$ & $\begin{array}{r} \\
+9.3\end{array}$ & 7 & $\begin{array}{l}\text { December } 10 \\
\text { (roup) }\end{array}$ & $\begin{array}{l}\mathbf{R}=0.235 \\
\mathbf{L}=0.200\end{array}$ & -175 \\
\hline & & & & 8 & $\begin{array}{l}\text { December } 21 \\
\quad \text { (roup) } \ldots .\end{array}$ & $\begin{array}{l}\mathrm{R}=0.590 \\
\mathrm{~L}=0.635\end{array}$ & +7.6 \\
\hline
\end{tabular}

Wild Duck

\begin{tabular}{|c|c|c|c|c|c|c|c|}
\hline 1 & $\begin{array}{r}\left(y^{\prime} g\right) \text { Novem- }\{ \\
\text { ber } 29 \ldots . . .\{\end{array}$ & $\begin{array}{l}\mathbf{R}=0.023 \\
\mathrm{~L}=0.027\end{array}$ & +17.4 & 2 & $\begin{array}{l}\text { December } 28 \\
\quad \text { (starved) } . . \\
\mathrm{R}=8.3 \times 3.2\end{array}$ & $\begin{array}{l}\mathbf{R}=0.040 \\
\mathrm{~L}=0.040 \\
\mathrm{~L}=8.6 \times 2\end{array}$ & $.5^{0.0}$ \\
\hline
\end{tabular}

${ }^{1}$ These cocks had been given a few injections of ovarian extract during the week preoeding the days of killing and autopsy. 


\section{SUMMARY}

The prevalence of atrophy of the right ovary in birds; the demonstrated differences in number of primordial germ cells in the two glands of the fowl; and the unequal-and oppositesize relations of the two adult gonads of the male, constitute a body of puzzling facts whose elucidation should contribute largely to our knowledge of the nature and basis of sexual difference.

The right testis of the pigeon is normally larger than the left.

In hybrid pigeons there are more exceptions to the normal size-relations of the two testes than in pure species. The number of the exceptions seems to increase with the degree of hybridization (width of the cross); there being fewer in specific hybrids than in generic hybrids.

The testes of pigeons suffer great reduction in size in diseaseparticularly in tuberculosis. It is probable that the right gland suffers greater reduction than the left. The left (persistent) gonad of the female does not suffer a similar reduction in tuberculosis. Season is plainly not the cause of the differences and reductions noted in pigeons.

The two testes of the pigeon are characteristically different in their dimensions. The left (like the left ovary) is thinner and more elongate. The right (represented in the female by atrophied ovary) is shorter and thicker.

In poultry the few data at hand fail to indicate a constant or decided predominance of size in either gland.

Cold Spring Harbor, L, I., N. Y. June, 1916

\section{LITERATURE CITED}

Adtuen, B. M. 1907 A statistical study of the sex cells in Chrysemys marginata. Anat. Rec., vol. 1, pp. 64-65. (Also Anat. Anz. vol. 30, pp. 391-399.)

ETzoLd, T. 1891 Die Entwicklung der Testikel von Fringilla domestica von der Winterruhe bis zum Eintritt der Brünst. Zeit. f. Wiss. Zool., vol. 52 , pp. $46-84$.

FIRKET, JEAN 1914 Recherches sur L'organogenese des glandes sexuelles chez les oiseaux. Arch. de. Biologie, T. 29.

HATAI, S. 1915 On the influence of exercise on the growth of the organs in the Albino rat. Anat. Rec., vol. 9.

Swirt, C. H. 1915 Origin of the definitive sex-cells in the female chick and their relation to the primordial germ cells. Am. Jour. Anat., vol. 18.

Tannengerg 1789 Spiclegium observationum eirca partes genitales masculas avium. Gottingen. 